\title{
Construction of Finite Cellular Automata Using the (123) - Avoiding Class of Aunu Permutation Pattern Application: In Game Of Chance
}

\author{
*Sadiq Shehu, *S.I. Abubakar *I. Zaid,*A.A. Ibrahim \\ *Department of Mathematics, Sokoto State University,P.M.B 2134, Sokoto.
}

\begin{abstract}
It was shown in Sadiq (2013) that succession parameters under the Aunu permutation patterns can be used as vertices of the graph model resulting from different transition of the automata scheme employed. This paper generates a graph model using the Aunu permutation patterns governed by some properties as embedded in method of construction of a typical game of chance scheme. A finite automata model was constructed from the game of chance using the (123) avoiding class of the Aunu permutation patterns.Furthermore, the paper illustrated some useful relationship between the field of automata theory and Combinatorics; it also highlights some important applications of the Aunu Permutation Patterns in graph theory.
\end{abstract}

Key words: Finite Automata, Permutation, Graph, In-degree, Out-degree, games

\section{Automata}

An automaton is a mathematical model of a very simplistic machine that jumps from one computational state to another depending on its current state on the last information bit it receives input. The automata is said to be deterministic if for each input the resulting computation is uniquely determined (Jeffrey, 2000). An automaton is commonly represented as a graph and every one of its computations is described by a path in the induced graph (Margolu, 1987).A cellular automaton (CA) is a mathematical "machine" or "Organism" that lends itself to some very remarkable and beautiful ideas. Among the most exciting aspects of this area of mathematics is that cellular automata arises from very basic mathematical principles. Though they are remarkably simple at the start; CAs has a variety of applications and can show up in places you would never expect from simple game of life, to interconnection network, computer simulation of biology to art (Ibrahim, 2008). Also is, at heart, a search for patterns and for a deep understanding of how and why they occur. It does Mathematics not matter if the patterns are in naturally occurring phenomena e.g., weather or population growth or in geometrical structures that we mentally impose upon reality to make sense of it E.g., triangles, circles, ellipses, or tetrahedral. These patterns may also be found in structures that we create for any number of reasons. Most of us find it more appealing to look for patters in this kind of image than in numbers themselves (Kathleen, 2001).

\subsection{Aunu permutation patterns}

The term Aunu patterns refers to a pairing scheme involving pairs of numbers associated with some precedence relation (Ibrahim, 2004; 2005). The governing conditions for generating these numbers are outline below.

The element are paired in order or precedence

$$
\lambda_{i} \lambda_{i} \ni i p j, i j \in S_{n}
$$

1. Where $S_{n}$ is a string of length $n$ while $\lambda_{i} \lambda_{i}$ are in the $i^{\text {th }}$ positions in the permutation pattern generated by the precedence relation $p$.

2. The precedence parameter (relation $\rho$ ) acts on the element to produce pairs that are related in such a way that an element and its first successor, element and its second successor, up to an element and its $n^{\text {th }}$ successor. Under the given condition, it is required that the $j^{\text {th }}$ partner shifts in position incrementally corresponding to the $n^{\text {th }}$ succession so that $j=i+1, i+2, \ldots \ldots \ldots \ldots, i+m$ where $m \leq n$

3. The numeration scheme involves doubt regarding the identity of the first element in the desired pair.

4. Absolute certainly is desired that by the end of the enumeration; the required pair, whichever it is, is achieved by denoting such a pattern as Aunu pattern.

\subsection{Applications to Game of Chance}

Until now, very particular sorts of automata have been considered using Aunu permutation pattern reported earlier (Ibrahim, 2012). 
It is important to know the transition rule from one state to another state as mentioned earlier, this paper will also consider elementary probability and a few applications of the ideas of CAs. The state and transition rules will appears different, as will the figures we draw.

An event here symbolizes the current fortune and each event is represented by a vertex or state. If the event $\mathrm{B}$ can be obtained from event A by a single coin - toss. We draw a direct line or arc from A to B and we call B a neighbor of $\mathrm{A}$. we call the resulting diagram a directed graph or digraph. So we use a digraph to picture the transition from one state to another. There are two kinds of states we want to identify. If a state has both "in coming" and "outgoing" arcs, we say this states is internal. If a state has only "incoming" arcs, we say this state is a boundary or absorbing state.

\subsection{Construction of game of chance using (123)-avoiding class of the Aunu permutationpatterns of cardinality five}

In what follows, we provide procedure on how to play a game of chance using the (123)-avoiding class of the Aunu permutation patterns of cardinality five.

Supposing you have $¥ 1$ and you need $\$ 5$. You can reach your goal by a fair game. You decide on the bold strategy: at each round you stake so much of your current fortune that you come as close to your goal as possible if you win. For example, if you would only need to bet $\$ 1$. You are bold enough to risk whatever it takes to get \#5, but you can never use more than you have at the moment the bold game can be translated into $\mathrm{G}_{1}$.

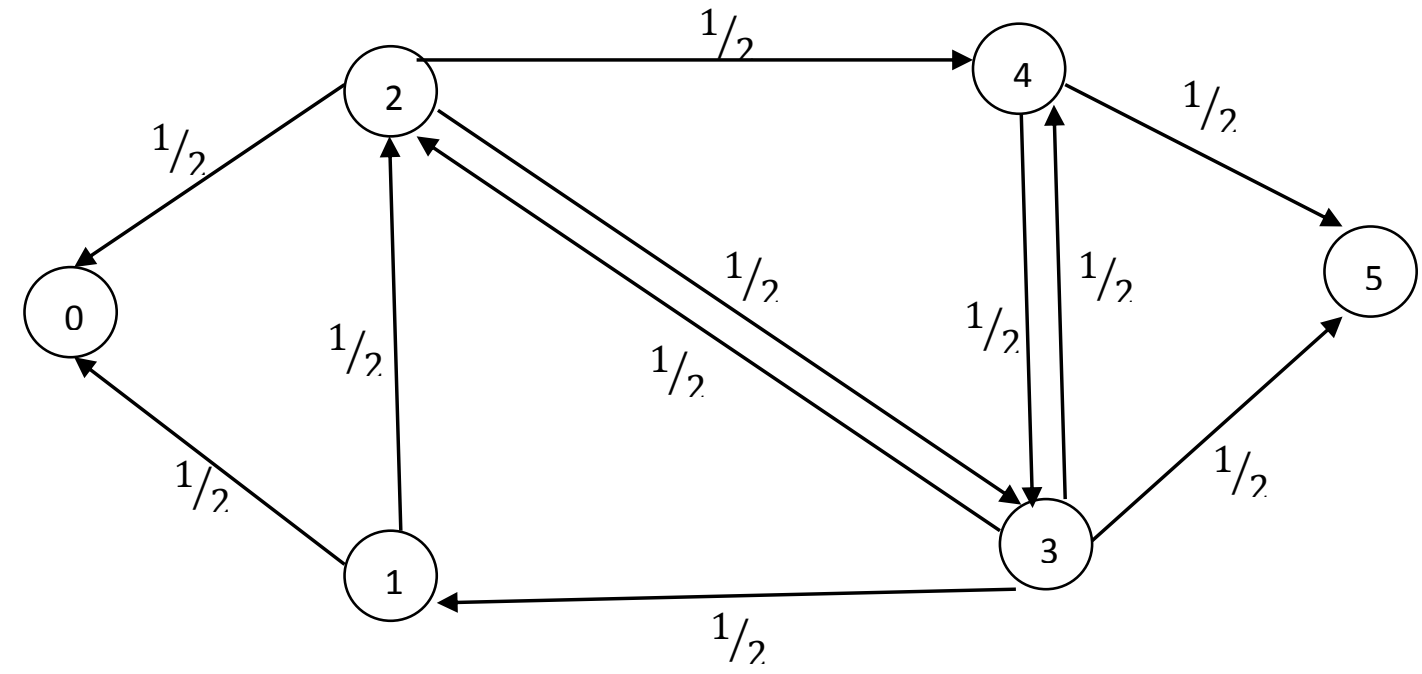

$\mathrm{G}_{1}$ : Game of chance using (123)-avoiding class of the Aunu permutation patterns of cardinality five.

A game of chance constructed using Aunu permutation pattern of cardinality five.

From $\mathrm{G}_{1}$ above each internal vertex there are two or more outgoing arcs, since we can either win or loss from this position. We have marked each arc with a " $1 / 2$ " to indicate a win or loss is equally likely for each individual bet you make. The direction of each arc is important for instance, the vertex labeled " 1 " indicates the start of the game, where you hold $\$ 1$. At the point you could bet just $\$ 1$ and either win (go to state "2") or lose (go to state "0"). Suppose you win, and are now in state 2. From here, you will bet your entire $\$ 2$. If you win this next bet, you will go to state 4: if you lost, you will (go to state "O'). If you are in state "3" you bet 2, if win you go to state "5", if you lose you go to state " 1 ", at the state " 3 " you bet 1 , if you win, you will go to state "4", If you lose you go to state " 2 ", if you are in state " 4 " you only need to get 1 to go to the accepting state i.e. state "5", if you lose you will go to state " 3 ".

\subsection{Construction of game of chance using (123)-avoiding class of the Aunu permutation patterns of cardinality seven}

In what follows, we provide procedure on how to play a game of chance using the (123)-avoiding class of the Aunu permutation patterns of cardinality seven.

Supposing you have $\$ 1$ and you need $\$ 7$ you can reach your goal by a pair of game. 


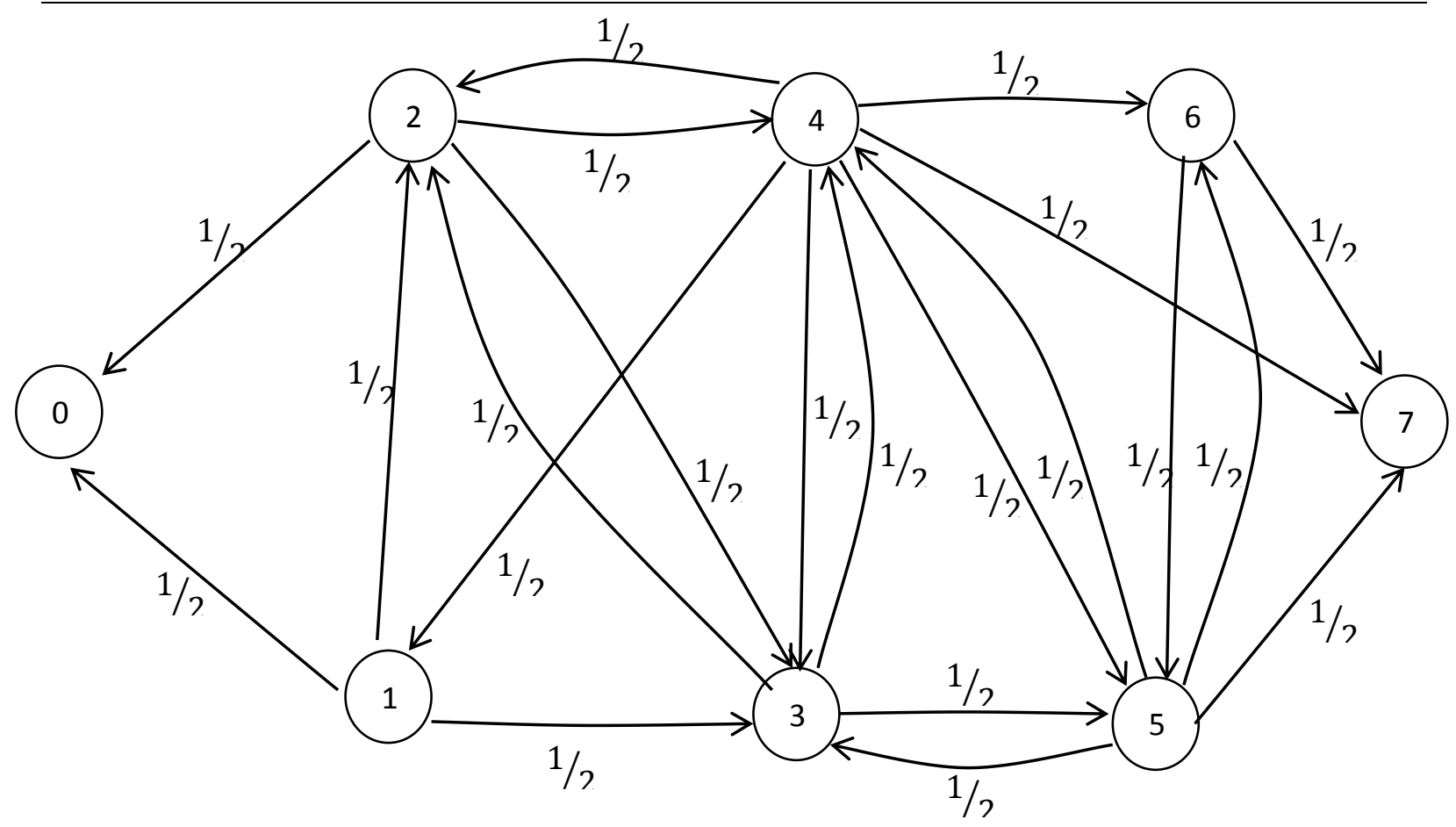

$\mathrm{G}_{2}$ : Game of chance using (123)-avoiding class of the Aunu permutation patterns of cardinality seven.

A game of chance constructed using Aunu permutation patterns of cardinality 7.

From the above digraph, the vertex labeled "1" indicates the start of the game, where you have $\$ 1$, at this point, you can just bet $\mathrm{N1}$ and either win (to go to state "2") or lose (go to state "O").

Suppose you win, you will go to state "2", from here you can bet your entire N2, if you win (go to state "4") or lose (go to state "O"). The game follows the same pattern/procedures as in graph model 2 above.

Theorem 1.7.1

(Euler's Degree-sum Theorem). The sum of the degrees of the vertices of a graph is twice the number of edges.

\subsection{Application of Euler's Degree-Sum Theorem on $\mathbf{G}_{1}$ and $\mathbf{G}_{2}$}

In what follows we provide some illustration of Euler's sum theorem on the game of chance $G_{1}$ and $G_{2}$ constructed by Aunu permutation patterns of cardinality five and seven respectively.

\subsubsection{Application in $\mathbf{G}_{1}$}

The in degrees and out degrees of the vertices of a digraph observed from Graph model3

\begin{tabular}{l|llllll} 
Vertex & 0 & 1 & 2 & 3 & 4 & 5 \\
\hline In degree & 2 & 1 & 2 & 2 & 2 & 2 \\
\hline Out degree & 0 & 2 & 3 & 4 & 2 & 0
\end{tabular}

1.8.2 Application in $\mathbf{G}_{2}$

The in degrees and out degrees of the vertices in the digraph observed from fig. $\mathrm{G}_{2}$

\begin{tabular}{l|llllllll} 
Vertex & 0 & 1 & 2 & 3 & 4 & 5 & 6 & 7 \\
\hline In degree & 2 & 2 & 3 & 3 & 3 & 3 & 2 & 3 \\
\hline Out degree & 0 & 2 & 3 & 4 & 6 & 4 & 2 & 0
\end{tabular}

\subsection{Conclusion}

This paper has illustrated some useful relationship between the field of automata theorem and Combinatorics, it also highlight some important application of the (123) avoiding class Aunu permutation patterns in graph theory. Similarly the game of chance was constructed on these generated models illustrating both the in degree and the out degrees of parameters of the model which result the application of Euler's Degree-sum theories.The paper also established some theoretical application of Aunu permutation patterns using for the first time the class of (123) - avoiding to form an automata game of chance. 


\subsubsection{Recommendation}

There are several other investigations that can be carried out on the constructed finite automata. An automata game of chance using (123) - avoiding class of Aunu permutation patterns for instance, further investigations can be carried out in other algebraic fields such as interconnection network and in topological spaces.

\section{References}

[1]. Ibrahim, A. A. (2004), "On wreath product of permutation Groups and algebraic theoretic properties of bara'at al-dhimmah models," $\mathrm{PhD}$ Thesis.

[2]. Ibrahim, A. A. (2005) "On the combination of succession in it - 5 element sample,"Abacus journal of mathematics Association on Nigeria Vol. 32 No. 2B 410-415

[3]. Ibrahim, A.A. and Audu, M.S. (2005), "Some group theoretic properties of certain class of (123) and (132) avoiding patterns of certain numbers; An enumeration scheme,"African Journal of Natural Science. Vol. 8:79 - 84

[4]. Ibrahim, A.A. and Audu, M.S. (2008), "A stable variety of Cayley Graphs for Efficient Interconnection networks,"proceeding of Annual conference of mathematical Association of Nigeria, 156-161.

[5]. Ibrahim, A.A. and M.S. Audu, (2008), "On stable variety of Cayley Graphs for Efficient interconnection networks," proceedings of Annual National conference of mathematical Association of Nigeria (MAN), $156-161$.

[6]. Jeffrey, D.Ullman, (2000), Introduction to Automata theory, languages and computation, Second Edition. 32: 456-510.

[7]. Kathleen, (2001). "Theory and Application of multiple Attractor Cellular Automata for fauit Diagnosis," international journal of Asian test Symposium. 321:388-392.

[8]. Sadiq S, et-al. (2013). Construction of finite Cellular Automata using (123)- avoiding Class of the Aunu Permutation Patterns: Application in Game of Life 